\title{
Developement of Crisp Package for Spallation Reaction Studies and its Utilization in ADS (Accelerator Driven System)
}

\author{
S. Anéfalos ${ }^{1}$, A. Deppman ${ }^{1}$, J. D. T. Arruda-Neto ${ }^{1,4}$, V. P. Likhachev ${ }^{1}$, \\ A. dos Santos ${ }^{2}$, P. R. P. Coelho ${ }^{2}$, S. B. Duarte ${ }^{3}$, and O. A. P. Tavares ${ }^{3}$ \\ 1) Instituto de Física, Universidade de São Paulo, IFUSP, Rua do Matão, Trav. R, 187, 05508-900, São Paulo, SP, Brazil \\ 2) Instituto de Pesquisas Energéticas e Nucleares - IPEN, Travessa R, 400, 05508-900, São Paulo, SP, Brazil \\ 3) Centro Brasileiro de Pesquisas Físicas - CBPF/MCT, Rua Dr. Xavier Sigaud, 150, 22290-180, Rio de Janeiro, RJ, Brazil \\ 4) Universidade de Santo Amaro, Rua Prof. Enéas de Siqueira Neto, 340, 04829-300, São Paulo, SP, Brazil
}

Received on 20 October, 2003

\begin{abstract}
The progress of nuclear physics had allowed a better understanding of nuclear structure and reaction mechanisms, but also enables important applications of nuclear energy in our modern way of life. We can point out those ones related to power generation from nuclear reactors, providing an almost inexhaustive power source due to the huge quantities of nuclear fuel existent in our planet, which guaranties its utilization for thousands of years. The main interest is the so-called Hybrid Reactors (ADS - Accelerator Driven System), in which the reactor has a sub critical core and the chain reaction is sustained by an external source that comes from spallation reactions induced by high energy protons (about $1 \mathrm{GeV}$ ) from an accelerator, producing a large number of secondary particles (mainly neutrons). For those applications, precise knowledge about nuclear structure and nuclear reaction characteristics is needed.
\end{abstract}

\section{Introduction}

Recently, the research groups from IFUSP and CBPF made a common effort to develop a computer program to calculate the intranuclear cascade proprieties and the nuclear evaporation process (MCMC/MCEF), present in all nuclear reactions with energies above few tens of $\mathrm{MeV}$, using Monte Carlo techniques. Some reaction channels were included in those programs, resulting in a more realistic representation of the processes involved, aiming Reactor Physics studies and also academic studies about hadrons and mesons properties in nuclear matter. The program has shown to be very useful to describe actinide and pre-actinide nuclear photofission reactions, demonstrating that the relevant processes that occur during the nuclear reaction are correctly calculated. In this work we will present some results obtained with this code and the comparison with experimental data.

The incineration of TRU is being considered by using fast neutrons from a spallation source [1], taking into account that the fission cross section is much higher than the capture cross section at these energies for most of TRU, and therefore transmuting long lived TRU in medium or short lived waste (FF). Besides the incineration of TRU and FF, a coupled system consisting of spallation source and a subcritical array of fuel, may have a positive gain of energy (net energy/energy to operate the accelerator). These facts has motivated innovative concepts of accelerator driven reactor in which thorium can be used as fuel [2].

\section{ADS - accelerator driven systems}

Rubbia et al [3] proposed a fast energy amplifier (EA) using a three stage modular cyclotron of $1 \mathrm{GeV}, 12.5 \mathrm{~mA}$ to induce spallation with protons in liquid lead target, Fig. 1. The fuel in a hexagonal array of pin-type elements forms the subcritical core surrounded the spallation source, and imbibed in liquid lead, which circulates by natural convection.

\section{MEA - modified energy amplifier}

We proposed some qualitative changes in the Rubbia's concept such as the use of more than one point of spallation, in order to reduce the requirement of proton energy and current of the accelerator beam, and mainly to make a flatter power density distribution [4]. The subcritical core, which in the Rubbia's concept is a hexagonal array of pins immersed in a liquid lead coolant, is replaced by a solid lead calandria with the fuel elements in channels cooled by Helium. This concept allows the utilization of a direct thermodynamic cycle (Brayton), which is more efficient than a vapor cycle. Gas cooled reactor technology is well established and more efficient from the thermodynamic point of view, allowing simplification and the utilization in high temperature process like hydrogen generation. The design constraints are a multiplication factor less than 0.97 in order to eliminate the 
need of safety rods, a radial peaking factor less than 2.0 and the maintenance of the lead between fuel elements solid.

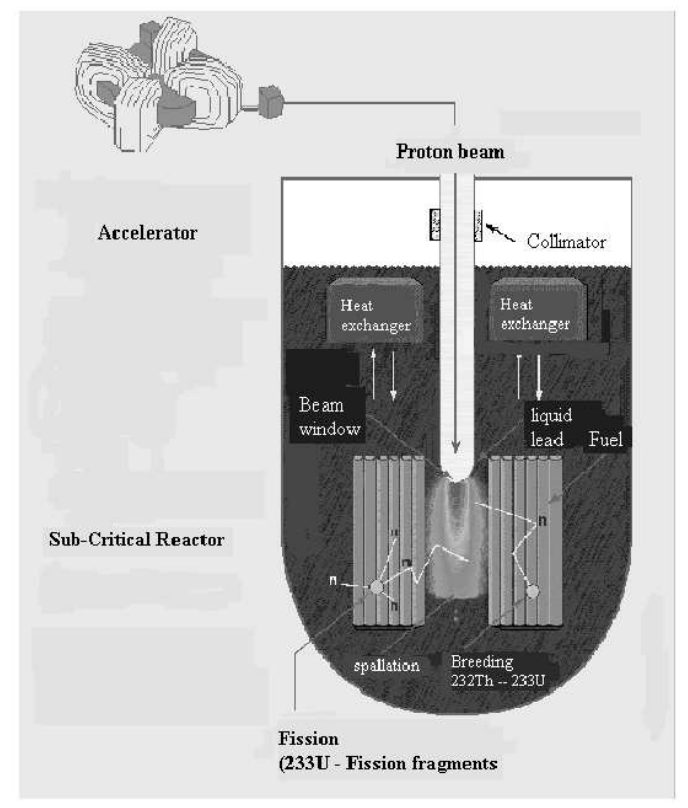

Figure 1. Schematic representation of Energy Amplifier proposed by Rubbia [4].

\section{Calculational metodology}

The methodology employed so far is shown in Fig. 2. It is based in the LAHET [5] and MCNP-4C [6] code systems. LAHET is the LANL version of the HETC Monte Carlo for the transport of protons. It's geometric transport capability is that of LANL's continuous energy neutron-photon Monte Carlo code MCNP-4C. The calculation methodology is made of two parts. In the first part LAHET performs the transport of protons and the subsequent characterization of the neutron and gamma sources arising from the spallation process in lead. For this purpose LAHET uses a Monte Carlo approach for the transport of protons through the lead target. It is in this program that CRISP can be coupled and became a better option for the intranuclear cascade and evaporation/fission calculations. The second part of the calculation methodology concerns the $\mathrm{k}_{\text {eff }}$ determination and the transport of neutrons and gamma through the fuel core and its surroundings. This task is accomplished by the continuous energy neutron-photon Monte Carlo code MCNP$4 \mathrm{C}$. Given the geometric description of the problem, the material composition for each zone, and a nuclear data library describing the neutron and/or gamma interaction with matter, MCNP-4C solves the coupled neutron-gamma transport equation and calculates several responses or tallies such as $\mathrm{k}_{e f f}$, total energy deposited by neutrons and gamma in lead as well as several other quantities of importance in reactor analyses. The nuclear data needed for MCNP-4C are generated by NJOY [7] accessing the ENDF/B-VI nuclear data file.

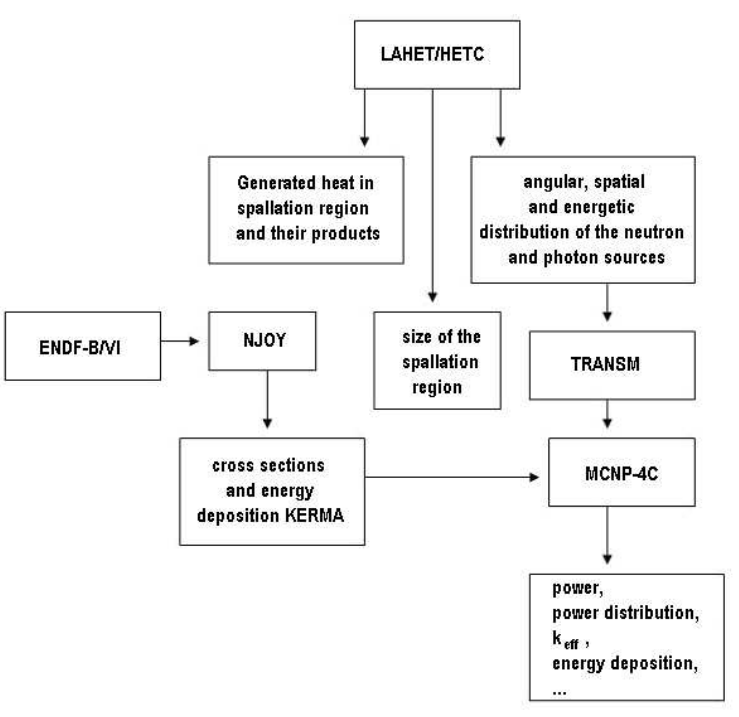

Figure 2. Methodology employed for the simulations.

\section{Spallation process}

Spallation is a nuclear reaction where a fast particle, such as a high-energy proton, bombards a heavy atomic nucleus, and some neutrons (and other particles) are "spalled" or knocked out. Given the energy of the incident particle it interacts in a first stage with the individual nucleons instead of the formation of a compound nucleus as in low energy nuclear reaction. The initial collision leads to an ejection of nucleons and pions, which still have enough energy to a cascade reaction (intranuclear cascades). After this phase, the nucleus is left in an excited state and goes to a ground state by evaporation of nucleons (mostly neutrons). Fragmentation (fission) of the nucleus may occur, as well as $(\mathrm{n}, \mathrm{xn})$ reaction in secondary stages. In short, the spallation reaction produces high energy secondary particles (neutrons, protons, mesons, gamma) which besides deposing a large amount of energy in the target and generating spallation product, escaping from the target, mainly neutrons, are the external source in the sub critical media. The most common used targets are $\mathrm{Pb}$ and $\mathrm{Bi}$.

\section{CRISP package (former MCMC/MCEF)}

The CRISP utilizes an algorithm that describes a many-body intranuclear cascade and evaporation/fission competition process, considering dynamic evaluation of the fermionic multicolisional process and the possibility of neutron, proton and alpha particle evaporation [8,9]. Fission process can also occur competing with the evaporation process. Other primary reaction channels are being included, such as the 


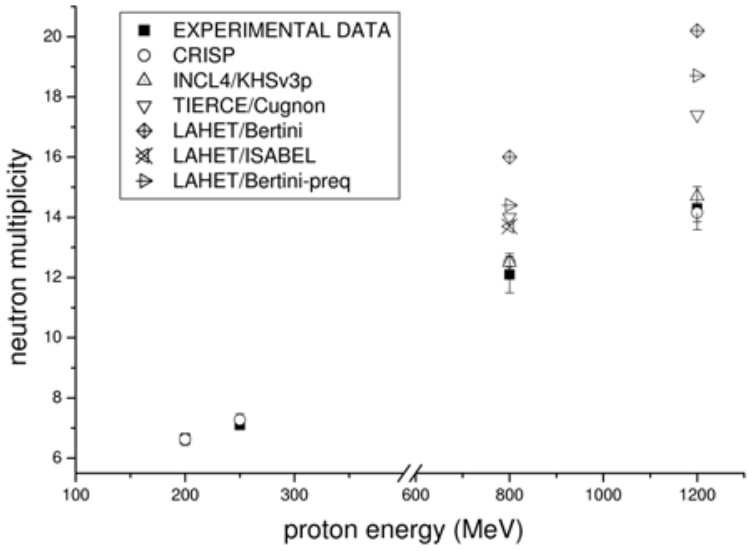

Figure 3. Neutron multiplicity in proton-induced reaction on $\mathrm{Pb}$ nuclei [12].

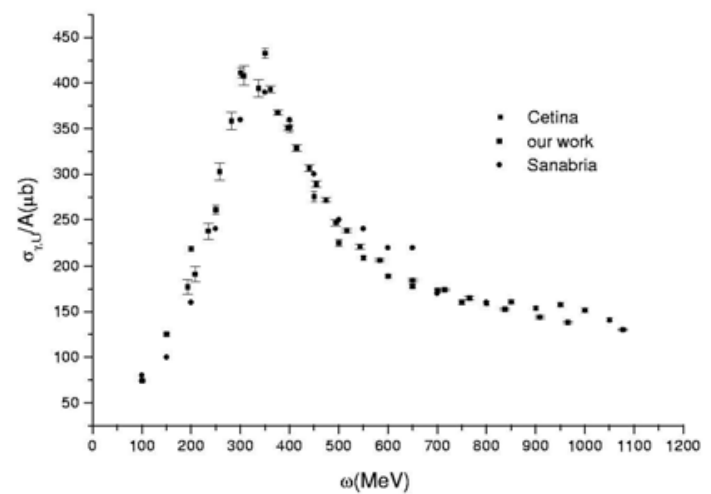

Figure 4. Photophission cross-sections per nucleon for $238 \mathrm{U}$.

formation of many nucleonic resonances and the shadowing effect. The Pauli blocking mechanism is already included. The nucleonic resonances occurs in $\gamma-\mathrm{N}$ and $\mathrm{p}-\mathrm{N}$ reactions, which can lead to short life particles $\left(10^{-23} \mathrm{~s}\right)$, and therefore resonant width, $\Gamma$, around $200 \mathrm{MeV}$. There are several resonances with their correspondent mass and half-life probabilities. The shadowing effect is based in the hypothesis of the vector dominance model, in which a photon with energy E $\gamma$ hitting a nucleus, can occupy, at the same time, the barephoton stage, electromagnetic stage and the hadronic stage. With the inclusion in our Monte Carlo code of the photon hadronization, resulting in the off-shell vector meson formation inside the nucleus, we will be able to calculate shadowing effects in a more realistic way than it can be done in the Glauber approximation.

\section{Preliminar results}

Although all these results are preliminary, they are very consistent with the available experimental data. Since the applicability of the CRISP package has a wide range of options, some results were chosen to describe the effectiveness of the code. We choose the neutrons multiplicity and the fissility results to exemplify its behavior. The first one is shown in
Fig. 3 and the second one is shown in Fig. 4. In Fig. 3 the neutron multiplicities in reactions induced by protons on $\mathrm{Pb}$ nuclei is compared with the predictions of our model and those from other models in a wide range of energy from a few hundreds of $\mathrm{MeV}$ [11] to $\mathrm{GeV}$ [12]. ISABEL cannot be run in LAHET above $1 \mathrm{GeV}$.

\section{Conclusions}

The CRISP package has been developed to describe intermediate energy nuclear reaction. It presents the possibility for studying gamma, proton and neutron induced reaction. It is currently being applied for photonuclear studies, ADS and Medical Physics. CRISP reproduces very well some experimental results related to neutron multiplicity, photofissility and others. The next step is to calculate the energetic and spatial distribution for different targets and compare the results with experimental data.

We are thankful to FAPESP, CNPq and CAPES (Brazilian agencies) for their support.

\section{References}

[1] C. Rubbia et al., Realistic Plutonium elimination scheme with fast energy amplifiers and Thorium-Plutonium fuel. CERN/AT/95-53 (ET), 1995.

[2] C. D. Bowman et al., Nuclear energy generation and waste transmutation using an accelerator-driven intense thermal neutral source. Nucl. Instr. And Methods A, 336-367, 1992.

[3] C. Rubbia et al., Conceptual design of a fat neutron operated high power energy amplifier. CERN/AT/95-44 (ET). 1995.

[4] S. Anéfalos, Um Conceito Alternativo de um Reator Hbrido (Conjunto Sub-Crítico Acoplado com Acelerador), $\mathrm{PhD}$ at IPEN-CNEN/SP, University of São Paulo, 2002.

[5] R. E. Prael and H. Linchtenstein, User Guide to LCS: The Lahet Code System. LA-UR-89-3014, Los Alamos National Laboratory, 1989.

[6] J. F.Briesmeiter, MCNP-4C- A General Monte Carlo NParticle Transport Code-Version 4C, Los Alamos National Laboratory, LA-13709-M (2000).

[7] R. E. Macfarlane et al., The N-JOY Nuclear Data Processing System: Users Manual, Los Alamos National Laboratory, LA-9303-M, Vol. I, ENDF-324 (1982).

[8] A. Deppman et al., Comp. Phys. Comm. 145, 385 (2002).

[9] S. B. Duarte et al.,Cluster approach to intranuclear cascade for heavy ions collisions, Phys. Rev. Lett. 49, 538 (1982).

[10] R. E. Prael and H. Lichtenstein, User guide to LCS: The LAHET Code System. Los Alamos National Laboratory Report, 1989. (LA-UR-89-3014).

[11] ICRU 2000 Nuclear Data for Neutron and Proton Radiotherapy and for Radiation Protection, International Commission on Radiation Units and Measurements Report 63 (2000).

[12] A. Boudard et al., Intranuclear cascade model for a comprehensive description of spalltion reaction data. Phys. Rev. C 66, 044615 (2002). 